\title{
Technical Note: Identification of CT Texture Features Robust to Tumor Size Variations for Normal Lung Texture Analysis
}

\author{
Wookjin Choi ${ }^{1}$, Sadegh Riyahi', Seth J. Kligerman², Chia-Ju Liu ${ }^{3}$, James G. Mechalakos ${ }^{1}$, Wei Lu${ }^{*}$ \\ ${ }^{1}$ Department of Medical Physics, Memorial Sloan Kettering Cancer Center, New York, USA \\ ${ }^{2}$ Department of Radiology, University of California at San Diego, San Diego, USA \\ ${ }^{3}$ Department of Radiology, Memorial Sloan Kettering Cancer Center, New York, USA \\ Email: *luw@mskcc.org
}

How to cite this paper: Choi, W., Riyahi, S., Kligerman, S.J., Liu, C.-J., Mechalakos, J.G. and Lu, W. (2018) Technical Note: Identification of CT Texture Features Robust to Tumor Size Variations for Normal Lung Texture Analysis. International Journal of Medical Physics, Clinical Engineering and Radiation Oncology, 7, 330-338. https://doi.org/10.4236/ijmpcero.2018.73027

Received: June 12, 2018

Accepted: August 4, 2018

Published: August 7, 2018

Copyright $\odot 2018$ by authors and Scientific Research Publishing Inc. This work is licensed under the Creative Commons Attribution International License (CC BY 4.0).

http://creativecommons.org/licenses/by/4.0/

\begin{abstract}
Normal lung CT texture features have been used for the prediction of radiation-induced lung disease (RILD). For these features to be clinically useful, they should be robust to tumor size variations and not correlated with the normal lung volume of interest, i.e., the volume of the peri-tumoral region (PTR). CT images of 14 lung cancer patients were studied. Different sizes of gross tumor volumes (GTVs) were simulated and placed in the lung contralateral to the tumor. 27 texture features [nine from intensity histogram, eight from the gray-level co-occurrence matrix (GLCM) and ten from the gray-level run-length matrix (GLRM)] were extracted from the PTR. The Bland-Altman analysis was applied to measure the normalized range of agreement (nRoA) for each feature when GTV size varied. A feature was considered as robust when its nRoA was less than the threshold (100\%). Sixteen texture features were identified as robust. None of the robust features was correlated with the volume of the PTR. No feature showed statistically significant differences $(\mathrm{P}<$ 0.05 ) on GTV locations. We identified 16 robust normal lung CT texture features that can be further examined for the prediction of RILD.
\end{abstract}

\section{Keywords}

Radiation-Induced Lung Disease, Normal Lung Texture, Radiomics, CT, Stereotactic Body Radiotherapy

\section{Introduction}

Stereotactic body radiotherapy (SBRT) has emerged as a standard therapy for early-stage non-small cell lung cancer (NSCLC), especially in patients who are 
considered poor surgical candidates. Despite its excellent cure rate, radiation-induced lung disease (RILD) is a frequent dose-limiting complication of SBRT [1] [2] [3]. Predicting the risk of RILD is very helpful for treatment planning and disease management [4] [5] [6].

Normal tissue complication probability (NTCP) models were proposed to predict the possibility of toxicity according to the dose [7] [8]. Dosimetric and clinical risk factors have been well established for the NTCP models for RILD, but they are limited regarding performance and generalizability [5] [9]. Recently radiomics particularly computed tomography (CT) texture features have been used to improve the prediction accuracy [10] [11]. CT texture features quantify the spatial patterns of tissue density, such as homogeneity, coarseness, and correlation of CT attenuation [12]. Therefore they can characterize the radiologic manifestations of RILD, which include ground-glass opacities, consolidation, and pleural effusions [4]. They have been used to predict radiation pneumonitis in the peri-tumoral region (PTR) [13].

It is important to identify which features are robust to assure the accuracy and generalizability of a prediction model. Previous robustness studies concerned about registration [10] [14] [15], test-retest [16] [17] [18], machine variability [17] [18] [19], and inter-observer variabilities on tumor delineation [16] [20]. For a feature to be clinically useful, it should also be robust (relatively invariant or unbiased) to tumor size variations and not correlated (non-redundant) with the normal lung volume of interest [21] [22]. We performed a simulation study to examine CT texture features extracted from the PTR in the lung.

\section{Methods}

We simulated different sizes of gross tumor volumes (GTVs) on free-breathing planning CT scans from 14 patients with lung cancer. This retrospective study was approved by our institutional review board. Fourteen patients treated with SBRT for non-small cell lung cancer were randomly identified. All CT scans were acquired with a Philips Big Bore Brilliance 16-slice CT scanner (Philips Healthcare; Andover, MA) using the same acquisition parameters: $120 \mathrm{kV}, 400$ $\mathrm{mAs}, 16 \times 1.5-\mathrm{mm}$ collimation, $3-\mathrm{mm}$ slice thickness, and approximately $1 \times 1 \times$ $3-\mathrm{mm}$ resolution. We excluded two patients who had multiple tumors on the right lung and the left lung. The remaining patients had a single primary lung tumor. We simulated various sizes of spherical GTVs in the normal lung contralateral to the tumor. The peri-tumor regions of the simulated GTVs were analyzed for texture feature robustness. Figure 1 shows the flowchart of the feature robustness analysis and the details are given in the following sections. The following image analysis was implemented using the Insight Segmentation and Registration Toolkit (ITK, National Library of Medicine; Bethesda, MD) [23].

\subsection{GTV Simulation}

For each patient, six spherical GTVs of various sizes $(10,20,30,40,50$, and 60 

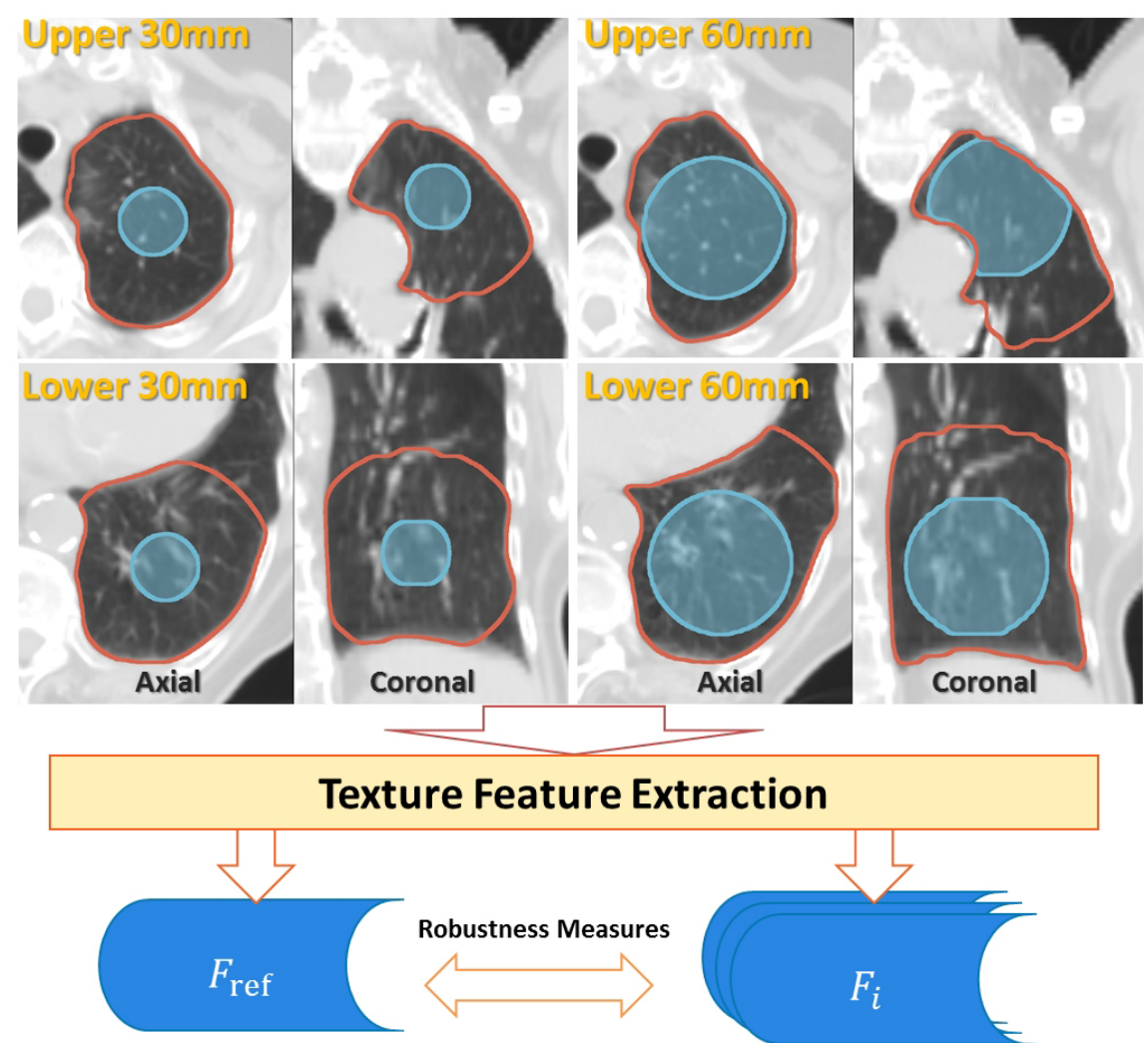

Figure 1. A flow chart for the robustness analysis of normal lung CT texture features extracted from the PTR (red contour excluding the simulated blue GTV). The mid-size (30 $\mathrm{mm}$ ) GTV was used as the reference tumor [ref].

$\mathrm{mm}$ diameters) were simulated in the contralateral lung. We placed all GTVs in the upper and lower lobes of the lung respectively, resulting in 12 GTVs for each patient and a total of 168 GTVs for all patients. The GTVs were placed to include homogeneous normal lung tissues at the same (upper or lower lobe) location for each patient while trying to exclude large blood vessels and underlying diseases (such as emphysema).

\subsection{Texture Feature Extraction}

A total of 27 texture features were extracted from each normal lung volume of interest, i.e., the PTR [13], defined as a $30 \mathrm{~mm}$ 3D expansion around the GTV (not including the GTV itself) excluding the chest wall and mediastinum. The texture features included nine intensity-histogram-based features and 18 spatial-frequency-based features (eight from the gray-level co-occurrence matrix [GLCM] [12] and ten from the gray-level run-length matrix [GLRM] [24] [25]).

Intensity-histogram-based texture features quantify the level and distribution of CT attenuations. The features were obtained by statistical measures of CT attenuation for all voxels within each PTR. These intensity features include first-order statistical measures and higher-order statistical measures.

Spatial gray-level co-occurrence and run-length matrixes estimate image properties related to higher-order statistics which consider the relationship among 
voxels or groups of voxels. The CT attenuation within each PTR was first normalized to the range from 0 to 255 . The texture features were then computed on the GLCM and GLRM of the normalized volumes. The mean value of each feature was computed over all 13 directions to obtain rotationally invariant features.

\subsection{Feature Robustness}

We chose the mid-size (30 mm diameter) GTV as the reference tumor [ref]. The robustness of a feature $F$ when GTV size varied was assessed using the normalized range of agreement (nRoA) with Bland-Altman method [14] [26]

$$
\begin{array}{r}
\text { nRoA }=\frac{95 \% \text { Limit upper bound }-95 \% \text { Limit lower bound }}{\mid \text { Mean } F \text { value } \mid} \times 100 \% \\
=\frac{\left(\mu_{F_{\text {bias }}}+1.96 \cdot \sigma_{F_{\text {bias }}}\right)-\left(\mu_{F_{\text {bias }}}-1.96 \cdot \sigma_{F_{\text {bias }}}\right)}{\left|\mu_{F}\right|} \times 100 \%
\end{array}
$$

with

$$
\mu_{F}=\frac{1}{\mathrm{nGTVs}} \sum_{i=1}^{\mathrm{nGTVs}} F_{i}
$$

where $F_{\text {bias } \_i}=F_{\text {ref }}-F_{i}$ was the difference of $F_{\text {ref }}$ ( $F$ calculated in the PTR of the [ref] tumor) and $F_{i}$ ( $F$ calculated in the PTR of the $I^{\text {th }}$ GTV); $\mu_{F_{\text {bias }}}$ and $\sigma_{F_{\text {bias }}}$ were the mean and the standard deviation of $F_{\text {bias } i}$ over all GTVs; and nGTVs and $\mu_{F}$ were the total number of GTVs (168) and the mean $F$ value across all patients, respectively. A smaller nRoA indicated smaller variation in $F$ as GTV size changed, and thus higher robustness of a feature. A feature was considered as robust if its $\mathrm{nRoA}$ was less than a threshold chosen around the nRoA of the volume of the PTR [10]. Furthermore, a feature was regarded as not correlated with the volume of the PTR when their correlation was lower than 0.70 .

\section{Results}

We considered a feature as a robust feature if its nRoA was smaller than the nRoA of the volume of the PTR. However, as shown in Figure 2, although the nRoAs of three features (GNU, Sum, and Kurtosis, Feature \#17-19) were smaller than the nRoA of the volume (\#20), we considered them as unrobust features. Because (1) there was a large gap in $n$ RoA between Inertia (\#16, 83.6\%, robust) and the three features, and (2) the nRoAs of the three features were very similar to the nROA of the volume (122.3\%). Based on these observations, we chose an nRoA threshold of $<100 \%$ to define a robust feature.

We identified 16 robust normal lung CT texture features extracted from the PTR whose nRoA was smaller than the threshold (100\%) as shown in Table 1. Most intensity histogram features were robust except maximum, sum and Kurtosis. All GLCM features were robust except cluster shade, cluster prominence, and Haralick's correlation. Both run emphasis features (SRE and LRE), and all 


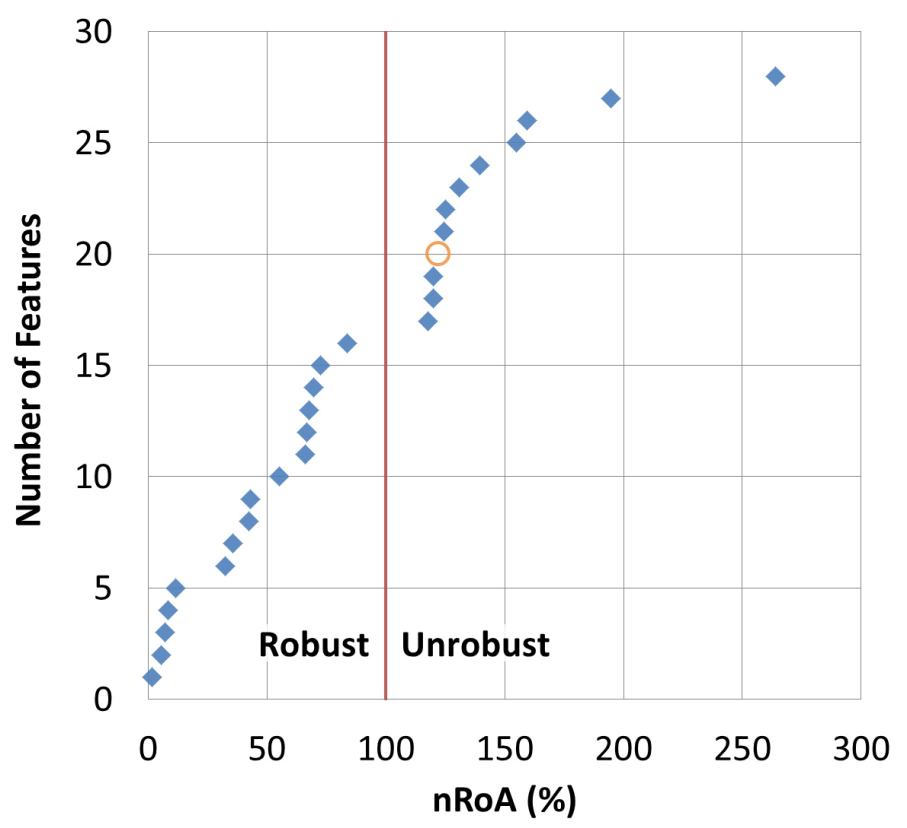

Figure 2. The cumulative graph of features vs. nRoA. Blue diamonds: texture features, orange circle: volume of the PTR, and red line: the threshold for robustness.

three high gray-level emphasis features (HGRE, SRHGE, and LRHGE) were robust. On the contrary, both nonuniformity features (GNU and RNU), and all three low gray-level emphasis features (LGRE, SRLGE, and LRLGE) were unrobust. Particularly, all three low gray-level emphasis features had large nRoAs (155\% - 264\%), indicating large variations when GTV size changed.

None of the robust features was correlated with the volume of the PTR (Table 1 ), suggesting that they can provide supplemental information to the volume. Two unrobust features (sum and RNU) were highly correlated with the volume of the PTR. No feature showed statistically significant differences $(\mathrm{P}<0.05)$ on GTV location (upper vs. lower lobe).

\section{Discussion}

Lung texture contains a distribution of both low attenuation and high attenuation tissues. Low attenuation predominates as a large portion of the lung volume consists of air within the airways and alveoli. However, a percentage of the lung is comprised of higher attenuation normal tissues including the vasculature and interstitium. Also, various pathologic states such as a tumor, pneumonia, hemorrhage, edema, and fibrosis lead to increased lung attenuation.

Most of the intensity and GLCM features are scale-invariant by definition, but some of them (e.g., maximum, kurtosis, CS, CP, and HC) were unrobust to GTV variations. Because, they were very sensitive to intensity variation affected by the volume change.

Most of the runs (same intensity straight lines) in the normal lung tissue are short low gray-level runs due to the proximal airway and alveoli which have high attenuation boundaries such as airway wall and interstitium. There are some 
Table 1. Normal lung CT texture features: normalized range of agreement (nRoA), correlation with the volume of the PTR, and P-value of the correlation.

\begin{tabular}{|c|c|c|c|}
\hline & nRoA (\%) & Correlation & P-value \\
\hline Volume of the PTR & 122.3 & 1.00 & $<0.05$ \\
\hline \multicolumn{4}{|l|}{ Robust Features } \\
\hline \multicolumn{4}{|l|}{ Intensity } \\
\hline Minimum & 7.0 & -0.32 & $<0.05$ \\
\hline Median & 5.6 & 0.06 & 0.25 \\
\hline Mean & 8.5 & 0.09 & 0.10 \\
\hline Variance & 67.6 & 0.30 & $<0.05$ \\
\hline Standard deviation (SD) & 35.6 & 0.33 & $<0.05$ \\
\hline Skewness & 42.3 & -0.36 & $<0.05$ \\
\hline \multicolumn{4}{|l|}{$G L C M$} \\
\hline Energy & 72.6 & -0.22 & $<0.05$ \\
\hline Entropy & 11.6 & -0.31 & $<0.05$ \\
\hline Correlation & 69.5 & -0.17 & $<0.05$ \\
\hline Inverse difference moment (IDM) & 32.3 & -0.08 & 0.13 \\
\hline Inertia & 83.6 & 0.03 & 0.57 \\
\hline \multicolumn{4}{|l|}{ GLRM } \\
\hline Short run emphasis (SRE) & 1.5 & 0.25 & $<0.05$ \\
\hline Long run emphasis (LRE) & 42.9 & -0.63 & $<0.05$ \\
\hline High gray-level run emphasis (HGRE) & 66.1 & 0.39 & $<0.05$ \\
\hline Short run high gray-level emphasis (SRHGE) & 66.7 & 0.39 & $<0.05$ \\
\hline Long run high gray-level emphasis (LRHGE) & 55.3 & 0.25 & $<0.05$ \\
\hline Mean of Robust Features & 41.8 & & \\
\hline \multicolumn{4}{|l|}{ Unrobust Features } \\
\hline \multicolumn{4}{|l|}{ Intensity } \\
\hline Maximum & 125.0 & -0.07 & 0.21 \\
\hline Sum & 119.9 & -0.85 & $<0.05$ \\
\hline Kurtosis & 120.1 & -0.40 & $<0.05$ \\
\hline \multicolumn{4}{|l|}{ GLCM } \\
\hline Cluster shade (CS) & 139.5 & 0.09 & 0.09 \\
\hline Cluster prominence $(\mathrm{CP})$ & 194.7 & 0.08 & 0.14 \\
\hline Haralick's correlation (HC) & 130.9 & 0.38 & $<0.05$ \\
\hline \multicolumn{4}{|l|}{ GLRM } \\
\hline Gray-level nonuniformity (GNU) & 117.8 & 0.62 & $<0.05$ \\
\hline Run-length nonuniformity (RNU) & 124.4 & 0.85 & $<0.05$ \\
\hline Low gray-level run emphasis (LGRE) & 159.6 & -0.24 & $<0.05$ \\
\hline Short run low gray-level emphasis (SRLGE) & 155.0 & -0.23 & $<0.05$ \\
\hline Long run low gray-level emphasis (LRLGE) & 264.0 & -0.32 & $<0.05$ \\
\hline Mean of unrobust features & 150.1 & & \\
\hline
\end{tabular}


short high gray-level runs from the vasculature, interstitium and various pathologic states as well as some long low gray-level runs from the distal airway. When the GTV size increased, long low gray-level runs within the PTRs were truncated into shorter runs. Therefore, all three low gray-level emphasis features (LRGE, SRLGE, and LRLGE) and both run-length distribution features (GNU and RNU) varied significantly due to different truncation effects of various-size GTVs. On the contrary, the high-attenuation tissues like small vasculature or interstitium had mainly short runs, the distribution of these short high graylevel runs was not significantly affected. Therefore, all three high gray-level emphasis features (HGRE, SRHGE, and LRHGE) had smaller variations (nRoAs = $66.1 \%, 66.7 \%$, and $55.3 \%$ ).

This study has a limited scope: examining the robustness of normal lung CT texture features when simulated tumor volume changes. As such it has several limitations: it was only a simulation study, the simulated spherical GTV were much simplified compared to the shapes of real tumors. In addition, no prediction model was constructed, and no real RILD case was studied. In the future, we will feed the identified robust texture features along with the conventional dose and clinical risk factors [8] [9], to prediction models in real RILD datasets.

\section{Conclusion}

We identified 16 robust lung CT texture features which were relatively invariant to tumor size variations and not correlated with the volume of the PTR. Particularly the three GLRM high gray-level emphasis features (HGRE, SRHGE, and LRHGE) can characterize the radiologic manifestations (increased lung attenuation) of pulmonary abnormalities. Hence these features can be further examined for the prediction of the RILD.

\section{Acknowledgements}

This work was supported in part by the NIH/NCI Grant No. R01 CA172638 and the NIH/NCI Cancer Center Support Grant P30 CA008748.

\section{Conflict of Interests}

The authors declare that there is no conflict of interests regarding the publication of this paper.

\section{References}

[1] Maebayashi, T., Ishibashi, N., Aizawa, T., Sakaguchi, M., Sato, T., Kawamori, J. and Tanaka, Y. (2016) Radiation Pneumonitis Changes over Time after Stereotactic Body Radiation Therapy for Lung Tumors: Post-Treatment Cavity (Sunny-Side-up Egg-Like) Changes. Anticancer Research, 36, 5563-5570. https://doi.org/10.21873/anticanres.11141

[2] Matsuo, Y., Shibuya, K., Nakamura, M., Narabayashi, M., Sakanaka, K., Ueki, N., Miyagi, K., Norihisa, Y., Mizowaki, T., Nagata, Y. and Hiraoka, M. (2012) DoseVolume Metrics Associated with Radiation Pneumonitis after Stereotactic Body 
Radiation Therapy for Lung Cancer. International Journal of Radiation Oncology * Biology ${ }^{*}$ Physics, 83, e545-e549. https://doi.org/10.1016/j.ijrobp.2012.01.018

[3] Yamashita, H., Takahashi, W., Haga, A. and Nakagawa, K. (2014) Radiation Pneumonitis after Stereotactic Radiation Therapy for Lung Cancer. World Journal of Radiology, 6, 708-715. https://doi.org/10.4329/wjr.v6.i9.708

[4] Choi, Y.W., Munden, R.F., Erasmus, J.J., Park, K.J., Chung, W.K., Jeon, S.C. and Park, C.K. (2004) Effects of Radiation Therapy on the Lung: Radiologic Appearances and Differential Diagnosis. Radiographics. A Review Publication of the Radiological Society of North America, Inc, 24, 985-998.

[5] Zhang, X.J., Sun, J.G., Sun, J., Ming, H., Wang, X.X., Wu, L. and Chen, Z.T. (2012) Prediction of Radiation Pneumonitis in Lung Cancer Patients: A Systematic Review. Journal of Cancer Research and Clinical Oncology, 138, 2103-2116. https://doi.org/10.1007/s00432-012-1284-1

[6] Takeda, A., Ohashi, T., Kunieda, E., Enomoto, T., Sanuki, N., Takeda, T. and Shigematsu, N. (2010) Early Graphical Appearance of Radiation Pneumonitis Correlates with the Severity of Radiation Pneumonitis after Stereotactic Body Radiotherapy (SBRT) in Patients with Lung Tumors. International Journal of Radiation Oncology ${ }^{*}$ Biology ${ }^{*}$ Physics, 77, 685-690. https://doi.org/10.1016/j.ijrobp.2009.06.001

[7] Emami, B., Lyman, J., Brown, A., Coia, L., Goitein, M., Munzenrider, J.E., Shank, B., Solin, L.J. and Wesson, M. (1991) Tolerance of Normal Tissue to Therapeutic Irradiation. International Journal of Radiation Oncology ${ }^{*}$ Biology ${ }^{*}$ Physics, 21, 109-122. https://doi.org/10.1016/0360-3016(91)90171-Y

[8] Bentzen, S.M., Constine, L.S., Deasy, J.O., Eisbruch, A., Jackson, A., Marks, L.B., Ten Haken, R.K. and Yorke, E.D. (2010) Quantitative Analyses of Normal Tissue Effects in the Clinic (QUANTEC): An Introduction to the Scientific Issues. International Journal of Radiation Oncology ${ }^{*}$ Biology ${ }^{*}$ Physics, 76, S3-S9.

https://doi.org/10.1016/j.ijrobp.2009.09.040

[9] Appelt, A.L., Vogelius, I.R., Farr, K.P., Khalil, A.A. and Bentzen, S.M. (2014) Towards Individualized Dose Constraints: Adjusting the QUANTEC Radiation Pneumonitis Model for Clinical Risk Factors. Acta Oncologica, 53, 605-612. https://doi.org/10.3109/0284186X.2013.820341

[10] Cunliffe, A., Armato, S.G., Castillo, R., Pham, N., Guerrero, T. and Al-Hallaq, H.A. (2015) Lung Texture in Serial Thoracic Computed Tomography Scans: Correlation of Radiomics-Based Features with Radiation Therapy Dose and Radiation Pneumonitis Development. International Journal of Radiation Oncology ${ }^{*}$ Biology ${ }^{*}$ Physics, 91, 1048-1056. https://doi.org/10.1016/j.ijrobp.2014.11.030

[11] Mattonen, S.A., Tetar, S., Palma, D.A., Louie, A.V., Senan, S. and Ward, A.D. (2015) Imaging Texture Analysis for Automated Prediction of Lung Cancer Recurrence after Stereotactic Radiotherapy. Journal of Medical Imaging (Bellingham), 2, 041010. https://doi.org/10.1117/1.JMI.2.4.041010

[12] Haralick, R.M., Shanmugam, K. and Dinstein, I. (1973) Textural Features for Image Classification. IEEE Transactions on Systems, Man, and Cybernetics, 3, 610-621.

[13] Palma, D.A., van Sornsen de Koste, J.R., Verbakel, W.F. and Senan, S. (2011) A New Approach to Quantifying Lung Damage after Stereotactic Body Radiation Therapy. Acta Oncologica, 50, 509-517. https://doi.org/10.3109/0284186X.2010.541934

[14] Cunliffe, A.R., Al-Hallaq, H.A., Labby, Z.E., Pelizzari, C.A., Straus, C., Sensakovic, W.F., Ludwig, M. and Armato, S.G. (2012) Lung Texture in Serial Thoracic CT Scans: Assessment of Change Introduced by Image Registration. Medical Physics, 39, 4679-4690. https://doi.org/10.1118/1.4730505 
[15] Cunliffe, A.R., Armato, S.G., Fei, X.M., Tuohy, R.E. and Al-Hallaq, H.A. (2013) Lung Texture in Serial Thoracic CT Scans: Registration-Based Methods to Compare Anatomically Matched Regions. Medical Physics, 40, 061906. https://doi.org/10.1118/1.4805110

[16] Balagurunathan, Y., Gu, Y., Wang, H., Kumar, V., Grove, O., Hawkins, S., Kim, J., Goldgof, D.B., Hall, L.O., Gatenby, R.A. and Gillies, R.J. (2014) Reproducibility and Prognosis of Quantitative Features Extracted from CT Images. Translational Oncology, 7, 72-87. https://doi.org/10.1593/tlo.13844

[17] Mackin, D., Fave, X., Zhang, L., Fried, D., Yang, J., Taylor, B., Rodriguez-Rivera, E., Dodge, C., Jones, A.K. and Court, L. (2015) Measuring Computed Tomography Scanner Variability of Radiomics Features. Investigative Radiology, 50, 757-765. https://doi.org/10.1097/RLI.0000000000000180

[18] Hunter, L.A., Krafft, S., Stingo, F., Choi, H., Martel, M.K., Kry, S.F. and Court, L.E. (2013) High Quality Machine-Robust Image Features: Identification in Nonsmall Cell Lung Cancer Computed Tomography Images. Medical Physics, 40, 121916. https://doi.org/10.1118/1.4829514

[19] Lo, P., Young, S., Kim, H J., Brown, M.S. and McNitt-Gray, M.F. (2016) Variability in CT Lung-Nodule Quantification: Effects of Dose Reduction and Reconstruction Methods on Density and Texture Based Features. Medical Physics, 43, 4854-4865. https://doi.org/10.1118/1.4954845

[20] Parmar, C., Rios Velazquez, E., Leijenaar, R., Jermoumi, M., Carvalho, S., Mak, R.H., Mitra, S., Shankar, B.U., Kikinis, R., Haibe-Kains, B., Lambin, P. and Aerts, H.J. (2014) Robust Radiomics Feature Quantification Using Semiautomatic Volumetric Segmentation. PLoS ONE, 9, e102107. https://doi.org/10.1371/journal.pone.0102107

[21] Choi, W., Riyahi, S. and Lu, W. (2016) SU-F-R-31: Identification of Robust Normal Lung CT Texture Features for the Prediction of Radiation-Induced Lung Disease. Medical Physics, 43, 3379-3380. https://doi.org/10.1118/1.4955803

[22] Choi, W., Riyahi, S., Liu, C.-J. and Lu, W. (2017) Robust Normal Lung CT Texture Features for the Prediction of Radiation-Induced Lung Disease. International Journal of Radiation Oncology ${ }^{\star}$ Biology ${ }^{\star}$ Physics, 99, S196-S197. https://doi.org/10.1016/j.ijrobp.2017.06.488

[23] Ibanez, L., Schroeder, W., Ng, L. and Cates, J. (2005) The ITK Software Guide: Kitware.

[24] Galloway, M.M. (1975) Texture Analysis Using Gray Level Run Lengths. Computer Graphics and Image Processing, 4, 172-179. https://doi.org/10.1016/S0146-664X(75)80008-6

[25] Tang, X. (1998) Texture Information in Run-Length Matrices. IEEE Transactions on Image Processing, 7, 1602-1609. https://doi.org/10.1109/83.725367

[26] Bland, J.M. and Altman, D.G. (2007) Agreement between Methods of Measurement with Multiple Observations per Individual. Journal of Biopharmaceutical Statistics, 17, 571-582. https://doi.org/10.1080/10543400701329422 